University of Rhode Island

DigitalCommons@URI

Mechanical, Industrial \& Systems Engineering

Faculty Publications

2011

\title{
Analytical and experimental study on the fluid structure interaction during air blast loading
}

\author{
Erheng Wang \\ Jefferson Townsend Wright \\ University of Rhode Island, jeffersonwright@gmail.com \\ Aun Shukla \\ University of Rhode Island, shuklaa@uri.edu
}

Follow this and additional works at: https://digitalcommons.uri.edu/mcise_facpubs

Terms of Use

All rights reserved under copyright.

\section{Citation/Publisher Attribution}

Wang, E., Wright, J., \& Shukla, A. (2011). Analytical and experimental study on the fluid structure interaction during air blast loading. Journal of Applied Physics, 110(11), 114901. doi: 10.1063/1.3662948 Available at: https://doi.org/10.1063/1.3662948

This Article is brought to you for free and open access by the Mechanical, Industrial \& Systems Engineering at DigitalCommons@URI. It has been accepted for inclusion in Mechanical, Industrial \& Systems Engineering Faculty Publications by an authorized administrator of DigitalCommons@URI. For more information, please contact digitalcommons-group@uri.edu. 


\section{Analytical and experimental study on the fluid structure interaction during air blast loading}

Erheng Wang, Jefferson Wright, and Arun Shukla

Citation: Journal of Applied Physics 110, 114901 (2011); doi: 10.1063/1.3662948

View online: https://doi.org/10.1063/1.3662948

View Table of Contents: http://aip.scitation.org/toc/jap/110/11

Published by the American Institute of Physics

\section{Articles you may be interested in}

Numerical simulation of the fluid-structure interaction between air blast waves and soil structure

AIP Conference Proceedings 1588, 293 (2014); 10.1063/1.4866963

Nonlinear compressibility effects in fluid-structure interaction and their implications on the air-blast loading of structures

Journal of Applied Physics 100, 063519 (2006); 10.1063/1.2349483

Blast Wave from a Spherical Charge

The Physics of Fluids 2, 217 (1959); 10.1063/1.1705911

Pressure signature and evaluation of hammer pulses during underwater implosion in confining environments The Journal of the Acoustical Society of America 140, 1012 (2016); 10.1121/1.4960591

Interactions between Blast Waves and V-Shaped and Cone-Shaped Structures

AIP Conference Proceedings 1376, 149 (2011); 10.1063/1.3651860

Underwater implosion of glass spheres

The Journal of the Acoustical Society of America 121, 844 (2007); 10.1121/1.2404921

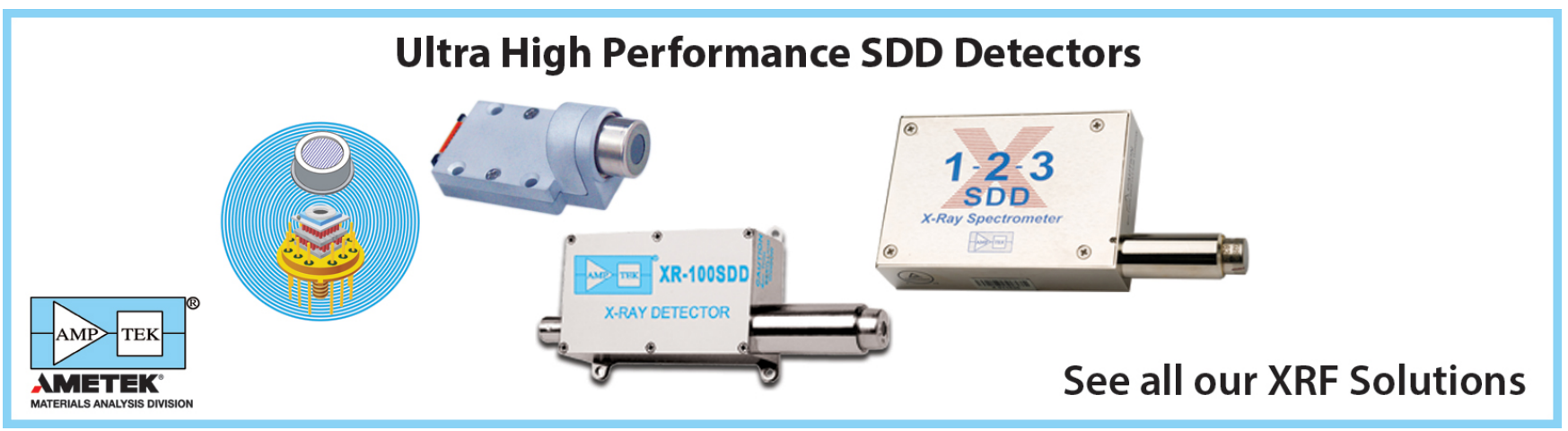




\title{
Analytical and experimental study on the fluid structure interaction during air blast loading
}

\author{
Erheng Wang, ${ }^{1}$ Jefferson Wright, ${ }^{2}$ and Arun Shukla ${ }^{2, a)}$ \\ ${ }^{1}$ Department of Aerospace Engineering, University of Illinois at Urbana-Champaign, Urbana, Illinois 61801 \\ ${ }^{2}$ Dynamic Photomechanics Laboratory, Department of Mechanical, Industrial and Systems Engineering, \\ University of Rhode Island, Kingston, Rhode Island 02881
}

(Received 29 June 2011; accepted 17 August 2011; published online 1 December 2011)

\begin{abstract}
A new fluid-structure interaction model that considers high gas compressibility is developed using the Rankine-Hugoniot relations. The impulse conservation between the gas and structure is utilized to determine the reflected pressure profile from the known incident pressure profile. The physical parameters of the gas such as the shock front velocity, gas density, local sound velocity, and gas particle velocity as well as the impulse transmitted onto the structure are also evaluated. A series of one-dimensional shock loading experiments on free standing monolithic aluminum plates were conducted using a shock tube to validate the proposed model. The momentum was evaluated using high speed digital imagery. The experimental peak reflected pressure, the reflected pressure profile, and the momentum transmitted onto the plate were compared with the predicted results. The comparisons show that the gas's compressibility significantly affects the fluid structure interaction behavior, and the new model can predict more accurate results than existing models. The effect of factors, such as the areal density of a plate and the peak incident pressure on momentum transfer are also discussed using the present model. Moreover, the maximum achievable momentum and the fluid structure interaction time are defined and calculated. (C) 2011 American Institute of Physics. [doi:10.1063/1.3662948]
\end{abstract}

\section{INTRODUCTION}

The fluid structure interaction behavior during blast loading plays a significant role in blast mitigation. Understanding the fluid structure interaction behavior, such as the maximum achievable impulse that can be imparted by a blast and the momentum transmitted to a specific target, helps in evaluating the blast performance of structures and consequently helps in the design of new structures with higher blast resistance.

The fluid structure interaction during a blast loading has been widely studied for many years. ${ }^{1,5-9}$ The classic solution for the interaction of a blast pulse with a solid plate was first derived by Taylor. ${ }^{1}$ He used the solution for a onedimensional wave impinging and reflecting upon a solid plate to compute the momentum transmitted onto the plate. The results showed that the momentum transmitted to a plate from a blast pulse is simply based on the density of the fluid, the wave speed, the blast decay time, and the areal density of the plate. This solution has been utilized to evaluate the blast resistance of sandwich composites with different core topologies. ${ }^{5-7}$ Because these researchers did not consider the non-linear compressibility of the fluid, the results for a blast loading in air, which has highly non-linear compressibility, have been questioned. ${ }^{10}$ Recently, Kambouchev et al. ${ }^{8,9}$ extended Taylor's model by considering the compressibility of the air. However, they derived the solution under the acoustic limit, which means that the propagation of pressure and density disturbances is governed by the linear wave

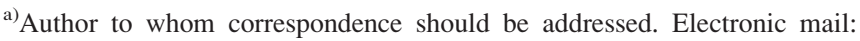
shuklaa@egr.uri.edu.
}

equation with a constant wave speed. For an intensive air blast loading, in which there is a noticeable difference between the incident and reflected shock wave velocities, ${ }^{11}$ further considerations are still needed. Glasstone, ${ }^{2}$ Baker et $a l .{ }^{3}$ and Smith et al. ${ }^{4}$ studied the effect of the air blast on a structure. They gave a semi-theoretical peak pressure of the reflected shock wave with normal incidence. Unfortunately, they could not evaluate the time histories of the reflected pressure as well as the reflected impulse. Baker et $\mathrm{al}^{3}$ recommended a rough estimation of the reflected impulse by assuming similarity between the time histories of a side-on overpressure and a normally reflected overpressure.

Some researchers ${ }^{12,13}$ have used pendulum experiments to estimate the impulse transmitted to the structures from a blast loading. This method can only estimate the final impulse transmitted to the structures and shows neither the impulse redistribution behavior nor the imparted impulse history during the blast event.

In this paper, a new fluid-structure-interaction model that considers the high compressibility of a fluid is proposed based on a one-dimensional gas-dynamic theory. The main goals of this paper are to:

(1) Develop a new one-dimensional fluid structure interaction model that considers the high gas compression during an air blast.

(2) Conduct a series of one-dimensional shock loading experiments on free-standing monolithic aluminum plates using a shock tube to validate the proposed model.

(3) Discuss the effects of parameters such as the areal density of the plate on the fluid structure interaction behavior and calculate the maximum achievable impulse. 
(4) Discuss possible errors in the proposed model and experiments.

\section{REVIEW OF THE FLUID-STRUCTURE INTERACTION MODELS}

Consider a uniform plane blast wave propagating with a constant velocity $U$ in a fluid of density $\rho_{f}$ and impinging normally upon a free-standing flat plate with an areal density $\rho_{\mathrm{s}}$. An approximate blast pressure profile is shown in Fig. 1. It has a very sharp jump at time $t=0$ with a very high overpressure above the background pressure (normally the atmospheric pressure). After the sharp jump, the pressure decays very quickly and even goes to a pressure level lower than the background pressure. The time at which the blast pressure is equal to background pressure is defined as positive time period $t_{+}$. The most common approximation of this pulse is expressed with an exponential decay profile,

$$
p(t)=p_{\text {peak }} e^{-\frac{t}{\theta}}, \quad 0 \leq t \leq \infty
$$

where, $p_{\text {peak }}$ is the peak pressure and $\theta$ is the time constant.

Based on this expression of the blast pressure profile, the free-field incident impulse per unit area of the blast is given as,

$$
I_{\text {incident }}=\int_{0}^{t_{+}} p(t) d t
$$

where, $t_{+}$is the positive time period of the pressure profile and can be related to the peak pressure $p_{\text {peak }}$ and the time constant $\theta$ by,

$$
\begin{gathered}
p_{\text {peak }} e^{-\frac{t_{+}}{\theta}}=p_{0} \\
\text { or, } \quad \theta=\frac{t_{+}}{\ln \left(p_{\text {peak }}\right)-\ln \left(p_{0}\right)}
\end{gathered}
$$

where, $p_{0}$ is the background pressure or the base pressure,

Throughout this paper, the traveling direction of the wave is defined as the positive $x$ direction, $x=0$ is chosen as the original position of the plate and the out-of-plane displacement of the plate is written as $w(t)$.

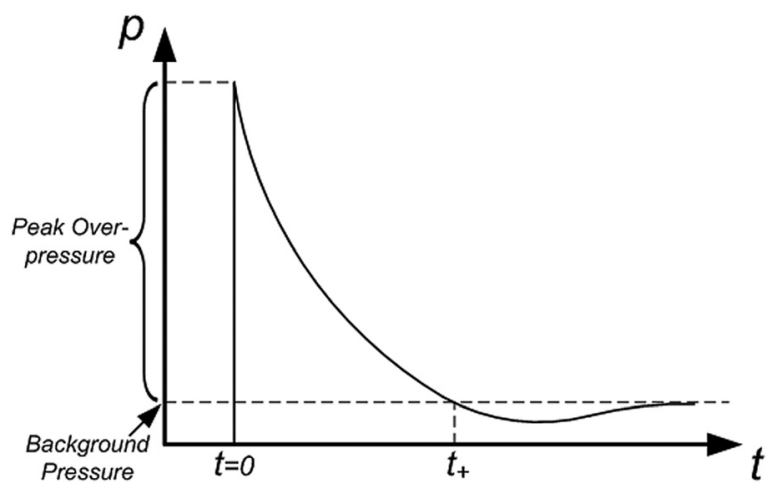

FIG. 1. Typical shock pressure profile.

\section{A. Taylor's model ${ }^{1,5-7}$}

In Taylor's model, the pressure applied on the plate can be separated into three parts: the pressure of the incoming wave $p_{I}$, the pressure of the reflected wave from a rigid wall $p_{r 1}$, and the pressure of the rarefaction wave $p_{r 2}$, due to the acceleration process of the plate. They can be expressed as,

$$
\begin{gathered}
p_{I}(t)=p_{\text {peak }} e^{-\left(t-\frac{x}{U}\right) / \theta} \\
p_{r 1}(t)=p_{\text {peak }} e^{-\left(t+\frac{x}{U}\right) / \theta} \\
p_{r 2}(t)=-\rho_{f} U \dot{w}\left(t+\frac{x}{U}\right),
\end{gathered}
$$

where $U$ is the shock wave front velocity.

Therefore, the pressure applied on the plate (at $x=0)$ is,

$$
p(t)=p_{I}(t)+p_{r 1}(t)+p_{r 2}(t)=2 p_{\text {peak }} e^{-\frac{t}{\theta}}-\rho_{f} U \dot{w}(t) .
$$

Application of the Newton's second law to the plate gives,

$$
\rho_{s} \ddot{w}(t)=2 p_{\text {peak }} e^{-\frac{t}{\theta}}-\rho_{f} U \dot{w}(t) .
$$

Considering the initial conditions $w(t)=\dot{w}(t)=0$ and defining a non-dimensional parameter $\psi=\left(\rho_{f} U \theta\right) / \rho_{s}$, the solution of Eq. (6) is,

$$
w(t)=\frac{2 p_{\text {peak }} \theta^{2}}{\rho_{s} \psi(\psi-1)}\left[(\psi-1)+e^{-\frac{\psi t}{\theta}}-\psi e^{-\frac{t}{\theta}}\right] .
$$

Then the pressure in Eq. (5) can be expressed as,

$$
p(t)=2 p_{\text {peak }} e^{-\frac{t}{\theta}}-\frac{2 p_{\text {peak }} \psi}{\psi-1}\left[e^{-\frac{t}{\theta}}-e^{-\frac{\psi t}{\theta}}\right] .
$$

Because the pressure should approach 0 at the time $t_{+}, t_{+}$ can be determined by substituting this condition into Eq. (8),

$$
t_{+}=\frac{\theta \ln (\psi)}{\psi-1} \text {. }
$$

The impulse transmitted into the specimen can be obtained,

$$
I_{\text {trans }}=\rho_{s} \dot{w}\left(t_{+}\right)=\zeta I_{\max },
$$

where, $I_{\max }$ is the maximum achievable impulse that is realized when the wave impinges upon a solid wall and $\zeta$ is the transmitted coefficient. They can be expressed as,

$$
\begin{gathered}
I_{\max }=\int_{0}^{\infty} 2 p_{\text {peak }} e^{-\frac{t}{\theta}} d t=2 I_{\text {incident }} \\
\zeta=\psi^{-\frac{\psi}{\psi-1}} .
\end{gathered}
$$

\section{B. Kambouchev's model ${ }^{8,9}$}

In Kambouchev's model, the compressibility of the fluid is considered by imposing the momentum conservation equation,

$$
\rho_{f} \frac{d u}{d t}=-\frac{d p}{d x}
$$

where, $u$ is the particle speed of the fluid. 
Under the acoustic limit condition, the pressure applied on the plate has the following form,

$$
p(t)=f(w-U t)+g(w+U t)
$$

where, $f$ and $g$ are arbitrary functions that should be determined.

Then by applying Newton's second law to the plate,

$$
\rho_{s} \ddot{w}(t)=f(w-U t)+g(w+U t) .
$$

Applying the momentum conservation Eq. (12) to the fluid,

$$
\rho_{f} \ddot{w}(t)=-f^{\prime}(w-U t)-g^{\prime}(w+U t) .
$$

Because function $f$ can be determined by the initial condition, one can obtain the governing equation by eliminating the unknown reflected wave $g$ from Eqs. (14a) and (14b),

$$
\rho_{s} \dddot{w}(t)+(\dot{w}+U) \rho_{f} \ddot{w}=-2 U f^{\prime}(w-U t) .
$$

Utilizing the conditions $\dot{w} \ll U$ and $f^{\prime}(w-U t) \approx f^{\prime}(-U t)$,

$$
\rho_{s} \dddot{w}(t)+\rho_{f} U \ddot{w}=-2 U f^{\prime}(-U t) .
$$

If $f$ is assumed to have an exponential decay profile as shown in Eq. (1) and initial conditions are then applied, one will obtain the same transmitted coefficient as expressed in Eq. (10) and (11) from Eq. (16). This indicates that the consideration of the compressibility of the fluid in this model can be ignored under the acoustic limit condition. Therefore this model is compatible with Taylor's model.

\section{THEORETICAL CONSIDERATIONS}

\section{A. Fluid structure interaction in an air blast loading}

Consider a planar shock wave impinging normally and uniformly upon an infinite free-standing monolithic plate with an areal density $m_{s}$. Figure 2 shows a typical shock wave loading process. A planar incident shock wave front is traveling from the left side to the right side (incident process, Fig. 2(a)). The right side of the incident wave front is undisturbed gas, whereas the left side of incident wave front is the high pressure gas driven by the incident shock wave. After the incident shock wave impinges on a static free-standing flat plate, a reflected planar shock wave is generated and travels from the right side to the left side (reflected process, Fig. 2(b)). The left side of the reflected wave front is still the high pressure gas driven by the incident shock wave, whereas the right side of the reflected wave front is now the gas that is disturbed by the reflected shock wave. Moreover, at this time the plate will move with a velocity that is equal to the particle velocity of the gas behind the reflected shock wave front. $U_{+}$and $U_{-}$are the velocities of the incident and the reflected shock wave fronts, respectively. The state of the gas can be defined using the following physical parameters:

(1) $p$ the pressure,

(2) $u$ the particle velocity,

(3) $c$ the sound velocity,

(4) $\rho$ the density.

The subscript 0 on the parameters denotes the initial state of the gas. Subscript 1 represents the state of the gas located behind the incident shock wave front, and this is defined as the incident state. Subscript 2 represents the state of the gas located behind the reflected shock wave front and this will be defined as the reflected state.

The incident pressure $p_{1}$ behind the incident shock wave front and reflected pressure $p_{2}$ behind the reflected shock wave front are all assumed to have exponential decay profiles. This will be shown to be true later from experimental data. They can be expressed as,

$$
\begin{array}{ll}
p_{1}(t)=p_{1 \_ \text {peak }} e^{-\frac{t}{\theta_{1}}}, & 0 \leq t \leq \infty \\
p_{2}(t)=p_{2 \_p e a k} e^{-\frac{t}{\theta_{2}}}, & 0 \leq t \leq \infty,
\end{array}
$$

where, $p_{1 \_ \text {peak }}$ and $p_{2 \_ \text {peak }}$ are the peak pressures for the incident and reflected pressure pulses, respectively. $\theta_{1}$ and $\theta_{2}$ are the time constants for the incident and reflected pressure. Furthermore, assume $t_{1+}$ and $t_{2+}$ are the positive time periods for the incident and reflected pressures.

From the shock wave loading processes described in Fig. 2, it can be seen that the plate is subjected to the reflected pressure, $p_{2}$, during the entire reflection process. Therefore, the impulse per unit area transmitted to the specimen, i.e., the final momentum per unit area of the specimen, is,

$$
I_{\text {impulse }}=\int_{0}^{t_{2+}}\left[p_{2}(t)-p_{0}\right] d t \text {. }
$$

Normally, the physical parameters of the undisturbed gas, $p_{0}$, $u_{0}, c_{0}$, and $\rho_{0}$ are known parameters. The pressure profile $p_{1}$ behind the incident shock wave front can be measured. However, other parameters, such as $p_{2}, u_{1}, u_{2}, c_{1}, c_{2}, \rho_{1}, \rho_{2}, U_{+}$, and $U_{-}$are all unknown parameters and are difficult to measure on-site. The absence of these physical parameters makes

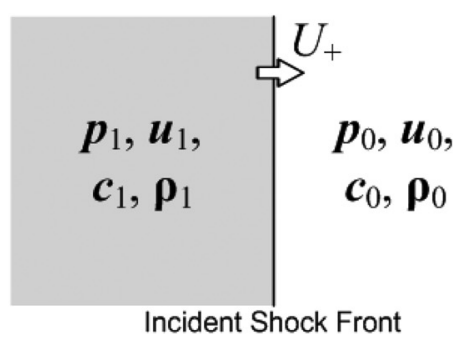

(a) Incident process

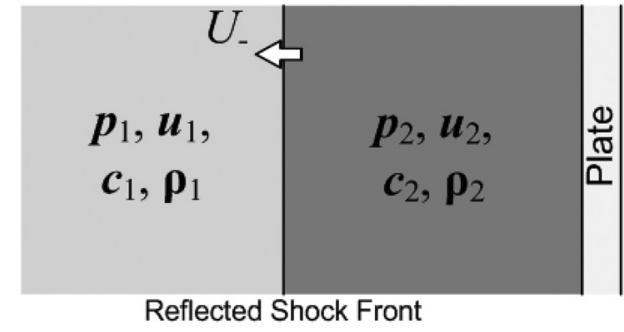

(b) Reflected process
FIG. 2. Sketch of the incident and the reflected shock processes. 
it difficult to evaluate the loading history of the plate. The following analysis proposes a methodology to calculate all of these unknown parameters based on the one-dimensional gas-dynamic theory.

\section{B. One-dimensional gas-dynamic theory for a shock wave in an ideal gas}

Because the gases during an air blast are all highly compressible, simple linear wave equations cannot be extended to apply to the shock wave loading process during an air blast. The gas-dynamic theory, which considers gas compressibility, is utilized to correlate the physical parameters of the gas in the present study.

The jump conditions for a shock wave front are as follows (using the incident shock wave process in Fig. 2(a) as an example): ${ }^{14}$

$$
\text { Conservation of mass: } \rho_{0} v_{0}=\rho_{1} v_{1}
$$

Conservation of momentum: $\rho_{0} v_{0}^{2}+p_{0}=\rho_{1} v_{1}^{2}+p_{1}$

Conservation of energy: $\frac{1}{2} v_{0}^{2}+e_{0}+p_{0} \tau_{0}=\frac{1}{2} v_{1}^{2}+e_{1}+p_{1} \tau_{1}$,

where, $v$ is the relative particle velocity with respect to the shock wave front. $v_{0}=u_{0}-U_{+}$and $v_{1}=u_{1}-U_{+}$.

The Rankine-Hugoniot relations with two modified forms can be derived from these jump conditions,

$$
\begin{gathered}
\frac{p_{1}}{p_{0}}=\left(1+\mu^{2}\right) M_{0}^{2}-\mu^{2} \\
\frac{p_{0}}{p_{1}}=\left(1+\mu^{2}\right) M_{1}^{2}-\mu^{2} \\
\left(1-\mu^{2}\right)\left(U_{+}-u_{0}\right)^{2}-\left(u_{1}-u_{0}\right)\left(U_{+}-u_{0}\right)=\left(1-\mu^{2}\right) c_{0}^{2} \\
\left(1-\mu^{2}\right)\left(U_{+}-u_{1}\right)^{2}-\left(u_{0}-u_{1}\right)\left(U_{+}-u_{1}\right)=\left(1-\mu^{2}\right) c_{1}^{2}
\end{gathered}
$$

where, $\mu^{2}=(\gamma-1) /(\gamma+1) ; \gamma$ is the adiabatic coefficient and $M$ is the Mach number, $M_{1}=\left|u_{1}-U_{+}\right| / c_{1}$ and $M_{0}=\left|u_{0}-U_{+}\right| / c_{0}$.

The jump conditions for the shock wave (Eqs. (19) (21)) and the modified Hugoniot relations (Eqs. (22) and (23)) compose the system of governing equations. Only three equations in this system are independent (Fig. 2(a)). The same procedure can be applied to the reflected process (Fig. 2b) by changing the subscript 0 to 1 , subscript 1 to 2 and subscript + to - . Thus, during a one-dimensional shock loading process (as in a shock tube experiment), there are six independent equations.

Though the shock wave is not a reversible process, the change of the parameters in the gas in front of and behind the shock wave front is a reversible, adiabatic process. This allows two more equations to correlate the pressure, density and sound velocity of the gas,

$$
\rho c^{2}=\gamma p
$$

$$
p=A \rho^{\gamma}
$$

where, $A$ is a constant for a reversible adiabatic process.

The proposed analysis is based on two hypotheses,

1. The velocity of the reflected shock wave front does not change during the shock wave loading process.

2. The physical properties of the gas, such as pressure and particle velocity, located on each side of the reflected shock wave front are uniform.

The feasibility and applicability of these two hypotheses have been discussed by Wang and Shukla. ${ }^{11}$

\section{IMPLEMENTATION OF THE PROPOSED FLUID STRUCTURE INTERACTION MODEL}

\section{A. Evaluation of the initial peak value of the physical parameters}

The expressions for the initial peak value of the parameters, $u_{1}, u_{2}, c_{1}, c_{2}, \rho_{1}, \rho_{2}, U_{+}$, and $U_{-}$, can be derived from the gas-dynamic equations in Sec. III B.

Through Eq. (22a), the incident shock wave velocity $U_{+}$ can be obtained as,

$$
U_{+}=c_{0} \sqrt{\frac{\left(p_{1 \_p e a k} / p_{0}\right)+\mu^{2}}{1+\mu^{2}}} .
$$

Using Eq. (23a), the peak particle velocity of the incident gas, $u_{1 \_ \text {peak }}$, can be calculated as,

$$
u_{1 \_ \text {peak }}=\frac{\left(1-\mu^{2}\right)\left(U_{+}^{2}-c_{0}^{2}\right)}{U_{+}},
$$

where the particle velocity $u_{0}$ of the undisturbed gas has been taken to be zero.

From Eq. (23b), the peak sound velocity of the incident gas, $c_{1 \_ \text {peak }}$, can be calculated as,

$$
c_{1 \_ \text {peak }}=\sqrt{\left(U_{+}-u_{1 \_p e a k}\right)^{2}+\frac{u_{1_{\perp p e a k}}\left(U_{+}-u_{1_{\perp} \text { peak }}\right)}{1-\mu^{2}} .}
$$

Using Eq. (19), the peak density of the incident gas, $\rho_{1 \_ \text {peak }}$, can be shown to be,

$$
\rho_{1 \text { peak }}=\left(\frac{u_{0}-U_{+}}{u_{1 \_p e a k}-U_{+}}\right) \rho_{0} .
$$

For the reflected process, Eqs. (19), (22a), (23a), and (23b) can be modified as,

$$
\begin{gathered}
\rho_{1}\left(u_{1}-U_{-}\right)=\rho_{2}\left(u_{2}-U_{-}\right) \\
\frac{p_{2}}{p_{1}}=\left(1+\mu^{2}\right)\left(\frac{u_{1}-U_{-}}{c_{1}}\right)^{2}-\mu^{2} \\
\left(1-\mu^{2}\right)\left(U_{-}-u_{1}\right)^{2}-\left(u_{2}-u_{1}\right)\left(U_{-}-u_{1}\right)=\left(1-\mu^{2}\right) c_{1}^{2} \\
\left(1-\mu^{2}\right)\left(U_{-}-u_{2}\right)^{2}-\left(u_{1}-u_{2}\right)\left(U_{-}-u_{2}\right)=\left(1-\mu^{2}\right) c_{2}^{2} .
\end{gathered}
$$


Then from Eq. (31), the reflected shock wave velocity $U_{-}$ can be calculated as,

$$
U_{-}=u_{1 \_p e a k}-c_{1 \_ \text {peak }} \sqrt{\frac{\left(p_{2 \_ \text {peak }} / p_{1 \_ \text {peak }}\right)+\mu^{2}}{1+\mu^{2}}} .
$$

From Eq. (32), the peak particle velocity of the gas behind the reflected shock wave, $u_{2 \_ \text {peak }}$, can be obtained as,

$$
u_{2 \_ \text {peak }}=u_{1 \_ \text {peak }}+\frac{\left(1-\mu^{2}\right)\left[\left(U_{-}-u_{1 \_p e a k}\right)^{2}-c_{1 \_p e a k}^{2}\right]}{U_{-}-u_{1 \_p e a k}} .
$$

From Eq. (33), the peak sound velocity of the reflected gas, $c_{2 \_p e a k}$, can be calculated as,

$$
c_{2 \text { peak }}=\sqrt{\left(U_{-}-u_{2 \text { peak }}\right)^{2}-\frac{\left(u_{1 \text { peak }}-u_{2 \text { peak }}\right)\left(U_{-}-u_{2 p e a k}\right)}{1-\mu^{2}}} .
$$

From Eq. (30), the peak density of the reflected gas, $\rho_{2 \_p e a k}$, can be calculated as,

$$
\rho_{2 \_ \text {peak }}=\left(\frac{u_{1 \_ \text {peak }}-U_{-}}{u_{2 \_ \text {peak }}-U_{-}}\right) \rho_{1 \text { peak }} .
$$

\section{B. Evaluation of the time history of the physical parameters}

To evaluate the time history of the physical parameters, one needs the incident pressure profile described by Eq. (17a), the reflected pressure profile described by Eq. (17b) and all of the initial peak values.

From Eq. (25), the density histories of the incident and reflected gases can be expressed as,

$$
\begin{aligned}
& \rho_{1}(t)=\left[\frac{p_{1}(t)}{p_{1 \_p e a k}}\right]^{\frac{1}{\gamma}} \rho_{1 \_ \text {peak }}=\rho_{1 \text { _peak }} \exp \left(-\frac{t}{\gamma \theta_{1}}\right) \\
& \rho_{2}(t)=\left[\frac{p_{2}(t)}{p_{2 \_ \text {_eak }}}\right]^{\frac{1}{\gamma}} \rho_{2 \_ \text {peak }}=\rho_{2 \_ \text {peak }} \exp \left(-\frac{t}{\gamma \theta_{2}}\right) .
\end{aligned}
$$

From Eq. (24), the sound velocity histories of the incident and reflected gases can be expressed as,

$$
\begin{aligned}
& c_{1}(t)=\left[\frac{\rho_{1}(t)}{\rho_{1 \text { peak }}}\right]^{\frac{\gamma-1}{2}} c_{1 \_p e a k}=c_{1 \_p e a k} \exp \left[-\frac{(\gamma-1) t}{2 \gamma \theta_{1}}\right] \\
& c_{2}(t)=\left[\frac{\rho_{2}(t)}{\rho_{2 \text { peak }}}\right]^{\frac{\gamma-1}{2}} c_{2 \_ \text {peak }}=c_{2 \_ \text {peak }} \exp \left[-\frac{(\gamma-1) t}{2 \gamma \theta_{2}}\right],
\end{aligned}
$$

then using Eqs. (31) and (32), the particle velocity histories of the incident and reflected gases can be expressed as,

$$
u_{1}(t)=U_{-}+c_{1} \sqrt{\frac{\left[p_{2}(t) / p_{1}(t)\right]+\mu^{2}}{1+\mu^{2}}}
$$

$$
u_{2}(t)=\frac{\left(1-\mu^{2}\right)\left\{\left[U_{-}-u_{1}(t)\right]^{2}-c_{1}^{2}\right\}}{U_{-}-u_{1}(t)}+u_{1}(t) .
$$

Note the signs of the particle velocities and shock wave velocities in Eqs. (26), (27), (34), (35), (42), and (43) indicate the direction of motion. In Fig. 2, a positive sign indicates the direction is the same as the propagating direction of the incident shock wave front and a negative sign indicates the direction is opposite that of the propagating direction of the incident shock wave front.

\section{Evaluation of the reflected pressure $p_{2}$}

From the analysis in Sec. IV A and IV B, all of the parameters can be expressed in terms of the reflected pressure $p_{2}$. If we can determine $p_{2}$, the proposed fluid structure interaction model is self-contained. The reflected pressure profile has been assumed to have an exponential decay profile described in Eq. (17b). To express this profile, three parameters need to be determined, peak reflected pressure, $p_{2 \_p e a k}$, the reflected time constant $\theta_{2}$ and the positive time period $t_{2+}$. There are only two unknown parameters; peak reflected pressure, $p_{2 \_p e a k}$, and the positive time period $t_{2+}$, due to the fact that the time constant $\theta_{2}$ can be calculated by Eq. (3),

$$
\theta_{2}=\frac{t_{2+}}{\ln \left(p_{2 \_ \text {peak }}\right)-\ln \left(p_{0}\right)} .
$$

\section{Determination of the peak reflected pressure $p_{2}$ peak}

The peak reflected pressure $p_{2 \_p e a k}$ is generated right after the shock wave front impinges upon the face of the plate. Because a shock wave front has a thickness of $10^{-8} \mathrm{~m}$ and a mach number larger than $1,{ }^{15,16}$ the time that the shock wave front passes the face plate has a value between $10^{-10}$ and $10^{-11} \mathrm{~s}$. A simple calculation shows that even for a peak reflected pressure as high as $1000 \mathrm{MPa}^{2}$ and an areal density of a plate as low as $1 \mathrm{~kg} / \mathrm{m}^{2}$ (about $0.4 \mathrm{~mm}$ aluminum foil), the velocity of the plate only has a value between $10^{-2}$ and $10^{-3} \mathrm{~m} / \mathrm{s}$, which can be thought of as negligible (zero). Notice the fact that the particle velocity $u_{2}$ of the gas behind the reflected shock wave front must be the same as the particle velocity of the plate in Fig. 2. ${ }^{1,14}$ Therefore, the peak reflected pressure can be calculated by assuming the particle velocity of the gas behind the reflected shock wave front is zero at the time when the shock wave just impinges upon the plate. Then from Eq. (35), one can obtain,

$$
0=u_{1 \_p e a k}+\frac{\left(1-\mu^{2}\right)\left[\left(U_{-}-u_{1_{\perp} \text { peak }}\right)^{2}-c_{1_{\text {_peak }}}^{2}\right]}{U_{-}-u_{1_{\text {ppeak }}}} .
$$

Substituting Eq. (34) into Eq. (45) gives,

$$
\begin{aligned}
p_{2_{-} \text {peak }}= & p_{1_{\text {_peak }}}\left\{\left[\frac{u_{1 \text { peak }}}{\left(1-\mu^{2}\right) c_{1 \_ \text {peak }}}+\sqrt{\frac{u_{1 \text { _peak }}^{2}}{\left(1-\mu^{2}\right)^{2} c_{1}^{2} \text { peak }}+4}\right]^{2}\right. \\
& \left.\times \frac{1+\mu^{2}}{4}-\mu^{2}\right\} .
\end{aligned}
$$


Then combining Eqs. (26) to (28) and (46) gives the value of the peak reflected pressure $p_{2 \_p e a k}$. In fact, this value is exactly the same as the well-known prediction proposed by Glasstone, ${ }^{2}$ Baker et al., ${ }^{3}$ and Smith et al., ${ }^{4}$ although the physical meaning of their peak reflected pressure is different than that discussed in this section. On the other hand, the value of the peak reflected pressure is independent of the loading process, and it is only related to the peak incident pressure. This means that plates with different areal densities can only change the time constant $\theta_{2}$ or the positive time period $t_{2+}$ when they are subjected to the incident shock wave with same incident pressure profile.

\section{Determination of the positive time period $t_{2+}$}

The positive time period $t_{2+}$ can be determined using the momentum conservation of the plate. As we know, at time $t=t_{2+}$, all of the impulse from the reflected pressure $p_{2}$, described in Eq. (18), has been transmitted onto the specimen. On the other hand, at time $t=t_{2+}$, the specimen will move with a velocity equal to the gas particle velocity $u_{2}$ behind the reflected shock wave front. Therefore the momentum conservation relation of the plate can be expressed as,

$$
\int_{0}^{t_{2+}}\left[p_{2}(t)-p_{0}\right] d t=m_{s} u_{2-\text { final }} .
$$

In this equation, $p_{0}$ and $m_{s}$ are known parameters. The final particle velocity $u_{2-\text { final }}$ of the plate at $\mathrm{t}=t_{2+}$ and the reflected pressure profile $p_{2}$ can be related through the analysis in preceding sections. Therefore, there is only one unknown parameter $t_{2+}$. It can be calculated from Eq. (47).

\section{Motion of the plate}

Because the load applied on the plates, i.e., the reflected pressure, has been determined through the preceding analysis, the motion of the plate can also be evaluated. Applying Newton's second law to the plate gives,

$$
p_{2}(t)=p_{2 \text { peak }} e^{-\frac{t}{\theta_{2}}}=m_{s} a_{s}(t),
$$

where, $a_{s}$ is the acceleration of the plate.

Now integrating the acceleration $a_{s}$ once and then again results in the velocity $v_{s}$ and displacement $d_{s}$ of the plate, respectively,

$$
\begin{gathered}
v_{s}(t)=\int_{0}^{t} a_{s}(t) d t=\int_{0}^{t} \frac{p_{2 \_ \text {peak }}}{m_{s}} e^{-\frac{t}{\theta_{2}}} d t=\frac{p_{2 \_ \text {peak }} \theta_{2}}{m_{s}}\left(1-e^{-\frac{t}{\theta_{2}}}\right) \\
d_{s}(t)=\int_{0}^{t} v_{s}(t) d t=\int_{0}^{t} \frac{p_{2 \_ \text {peak }} \theta_{2}}{m_{s}}\left(1-e^{-\frac{t}{\theta_{2}}}\right) d t \\
=\frac{p_{2 \_ \text {peak }} \theta_{2}}{m_{s}}\left(t+\theta_{2} e^{-\frac{t}{\theta_{2}}}-\theta_{2}\right) .
\end{gathered}
$$

\section{SHOCK WAVE LOADING EXPERIMENTS ON FREE-STANDING MONOLITHIC PLATES}

Shock tubes have been widely used for simulating blast loading as they can generate a tailored shock wave with a planar wave front. ${ }^{17-21}$ Because the shock wave front generated by a shock tube is flat, the shock wave loading process upon a flat plate can be considered a one-dimensional process. Moreover, there are several parameters that can be measured directly from shock tube experiments, such as the incident pressure profile, $p_{1}$, the reflected pressure profile, $p_{2}$, the incident shock wave velocity, $U_{+}$, and the reflected shock wave velocity, $U_{-}$. Therefore they can be used to verify the proposed fluid structure interaction model. In this study, a series of shock wave loading experiments on freestanding monolithic plates was carried out using a shock tube apparatus.

\section{A. Experimental setup}

The experimental setup used in this study is shown in Fig. 3. A shock tube apparatus was utilized to generate a planar shock wave with a controlled overpressure level. ${ }^{11,17,18,20,21}$ The muzzle end of the shock tube with the locations of the pressure transducers (PCB CA102B) is shown in Fig. 4. The inner diameter of the muzzle is $0.0762 \mathrm{~m}$, which is also the diameter of the loading area. Two pressure transducers are mounted at the end of the muzzle section to measure the pressure profile. The distance between the two transducers is $0.16 \mathrm{~m}$ and the distance between transducer 2 and the end of the muzzle is $\sim 0.02 \mathrm{~m}$.

A high speed digital camera, Photron SA1, was used to capture the real time side-view position of the plate. The axis of the camera lens is perpendicular to the axis of the shock tube, i.e., the moving direction of the plate. The distance between the camera and the plate was chosen to be approximately $2 \mathrm{~m}$, which is more than 20 times that of the plate's dimension $(\sim 0.1 \mathrm{~m})$ and 10 times that of the plate displacement in the images $(\sim 0.2 \mathrm{~m})$, to avoid image distortion during plate propagation.

\section{B. Material and specimen}

The circular plate specimens were fabricated using 6061 T6 Aluminum. The diameter of the flat circular plate

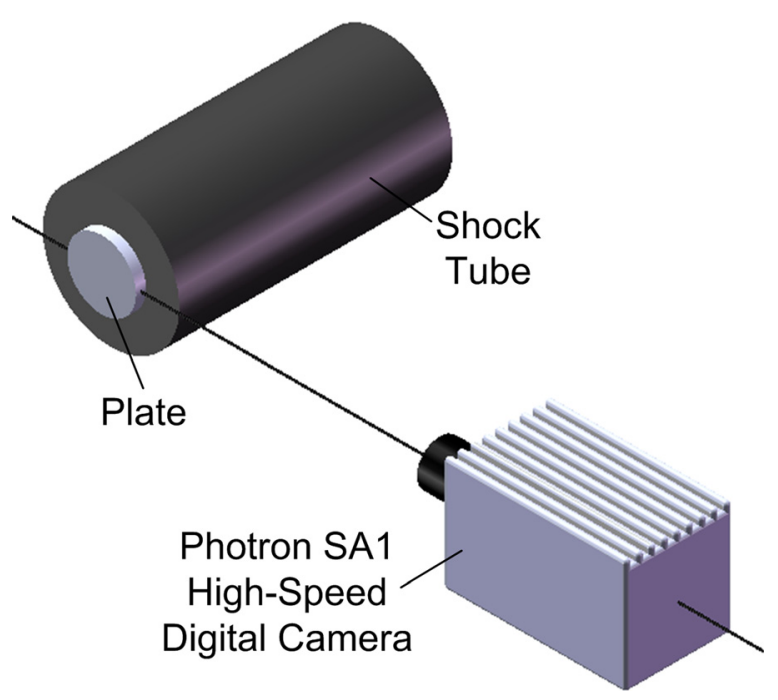

FIG. 3. (Color online) Experimental setup. 


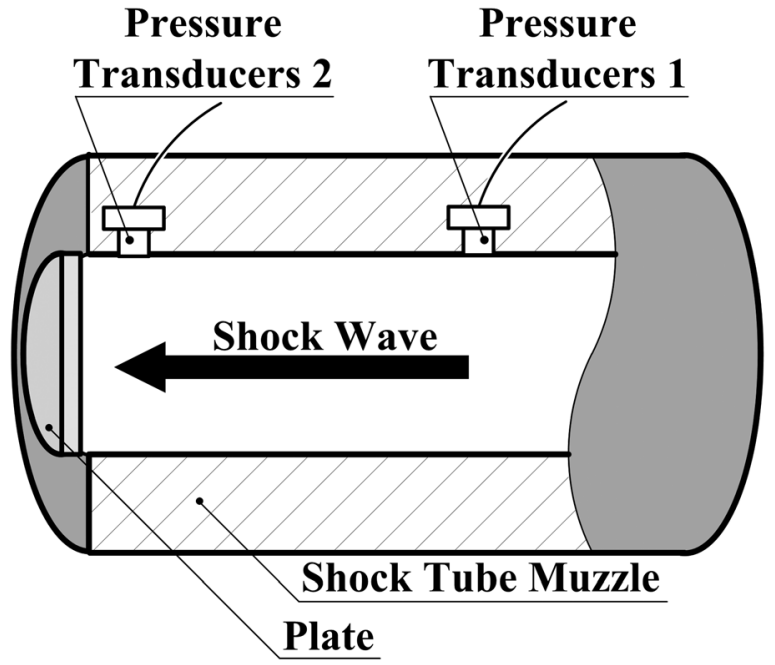

FIG. 4. Details of the muzzle end.

specimen was $0.0777 \mathrm{~m}$, which was slightly bigger than the inner diameter of the shock tube $(0.0762 \mathrm{~m})$, to ensure that the plate covers the entirety of the muzzle. The thickness of the specimens was $0.0064 \mathrm{~m}$ and its areal density was $17.1 \mathrm{~kg} / \mathrm{m}^{2}$.

\section{Experimental procedure}

During the experiments, the circular specimens were placed right in front of the muzzle end of the shock tube (as shown in Figs. 3 and 4). The specimens were then subjected to shock wave loadings with four different incident peak pressures: $0.44,0.76,1.03$, and $1.35 \mathrm{MPa}$, respectively. The experimental and the simulated (fitted) pressure profiles for different peak pressures are shown in Fig. 5. The time constants $\theta_{1}$ and positive time periods $t_{1+}$ for each pressure profile are listed in Table I.

For each shock wave level, two experiments were conducted for the purpose of verifying data repeatability. The high-speed digital camera was set to a framing rate of 40000 fps and an image resolution of $512 \times 256$ pixels.

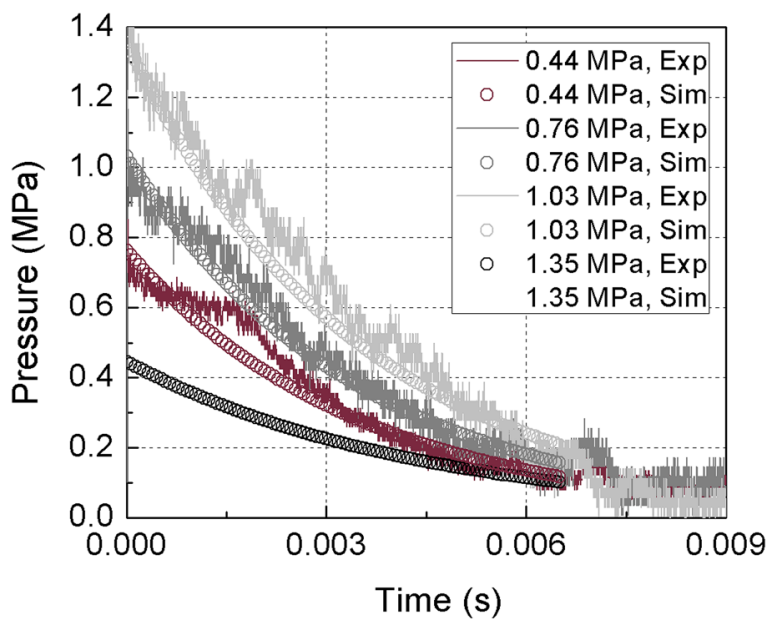

FIG. 5. (Color online) Incident pressure profiles with different incident peak pressure.
TABLE I. Parameters used for fitting the incident pressure profiles.

\begin{tabular}{lcc}
\hline \hline $\begin{array}{l}\text { Peak incident } \\
\text { pressure } \\
(\mathrm{MPa})\end{array}$ & $\begin{array}{c}\text { Positive time } \\
\text { period } t_{1+} \\
(\mathrm{s})\end{array}$ & $\begin{array}{c}\text { Time } \\
\text { constant } \theta_{1} \\
(\mathrm{~s})\end{array}$ \\
\hline 0.44 & 0.0065 & 0.0044 \\
0.76 & 0.0070 & 0.0035 \\
1.03 & 0.0080 & 0.0034 \\
1.35 & 0.0090 & 0.0034 \\
\hline \hline
\end{tabular}

\section{Evaluation of the momentum of the plate from high-speed images}

A typical high-speed side-view image during a shock wave loading process is shown in Fig. 6. Curve fitting methods, such as cubic spline curve fitting, can be used to pickup the position of the front face of the specimen. An example of the 7-point cubic spline curve fitting is shown in Fig. 6. Because the specimen did not show any compression during the shock wave loading process, this curve can be used to represent the position of the specimen. Then the displacement profile of each point on the specimen can be obtained by correlating the position of the specimen in each image to that in the image at time $t=0$. The differential of this displacement profile with respect to the time gives the velocity profile. The momentum of the plate can be evaluated from the velocity profile and the areal density of the plate. It can be expressed as:

$$
\text { Horizontal momentum: } I_{x}^{\text {plate }}=\oint_{S} m_{s} u_{x} d s \text {, }
$$

where, $u_{x}$ is the $x$ direction (horizontal) velocity and $d s$ is the areal element of the plate.

\section{RESULTS AND DISCUSSION}

\section{A. Experimental results}

The reflected pressure profiles measured by the pressure transducer closest to the muzzle end are shown in Fig. 7. The

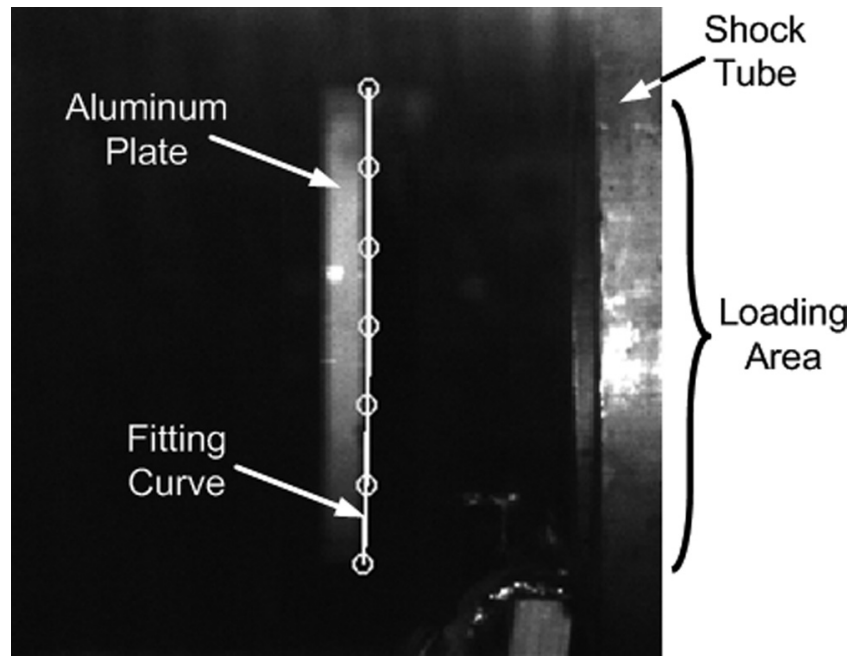

FIG. 6. A typical side-view image and curve fitting of the front face. 


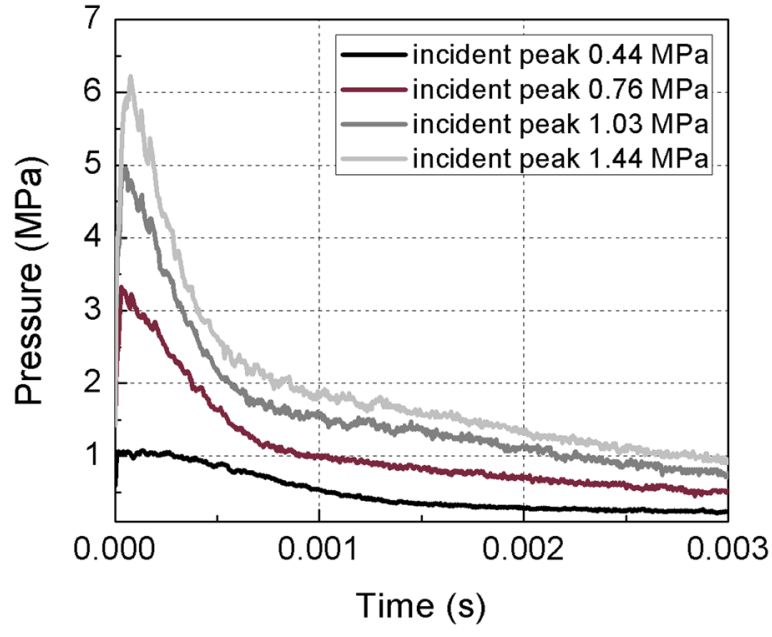

FIG. 7. (Color online) Experimental reflected pressure profiles.

high speed side-view images of the circular plates at different incident shock levels are shown in Fig. 8.

The momentum of the plates obtained by the method described in Sec. V D and the impulse of the reflected pressure (Eq. 18) are shown in Fig. 9. It can be seen that the momentum of the plates agrees with the impulse of the reflected pressure very well. This verifies that the momentum is conserved during the loading process as described by Eq. (47). Because the high speed side-view images can only be recorded for a short time period (generally less than $1 \mathrm{~ms}$ ), the impulse of the reflected pressure can be used to determine the momentum of the plates for longer time periods.

\section{B. Comparison of experimental and analytical results}

\section{Input parameters}

The parameters needed in the analytical model, such as the pressure, density, sound velocity and particle velocity of the undisturbed gas in front of the incident shock wave front, are listed in Table II.

\section{Reflected pressure profile}

The experimental and analytical results of the peak reflected pressure as a function of the incident pressures are shown in Fig. 10. The peak reflected pressure determined by Eq. (5) at time $t=0$ from Taylor's model is also shown in this figure. It can be seen that the experimental data follow the predicted trend very well. Taylor's model gives much lower prediction results. The model prediction has also been compared with the experimental data for various other materials tested in our lab and the results are shown in Fig. $11 .^{22}$ It is evident that the reflected peak pressure only depends on the incident peak pressure, and the compressibility of the gas highly affects the results.

The reflected pressure profile obtained from the experiment, Taylor's model (Eq. (5)) and the present model are shown in Fig. 12. For simplification, only the results of $1.03 \mathrm{MPa}$ peak incident shock pressure are provided. It can be seen that at the beginning (before $700 \mu \mathrm{s}$ ), the prediction from Taylor's model is much lower than the experimental
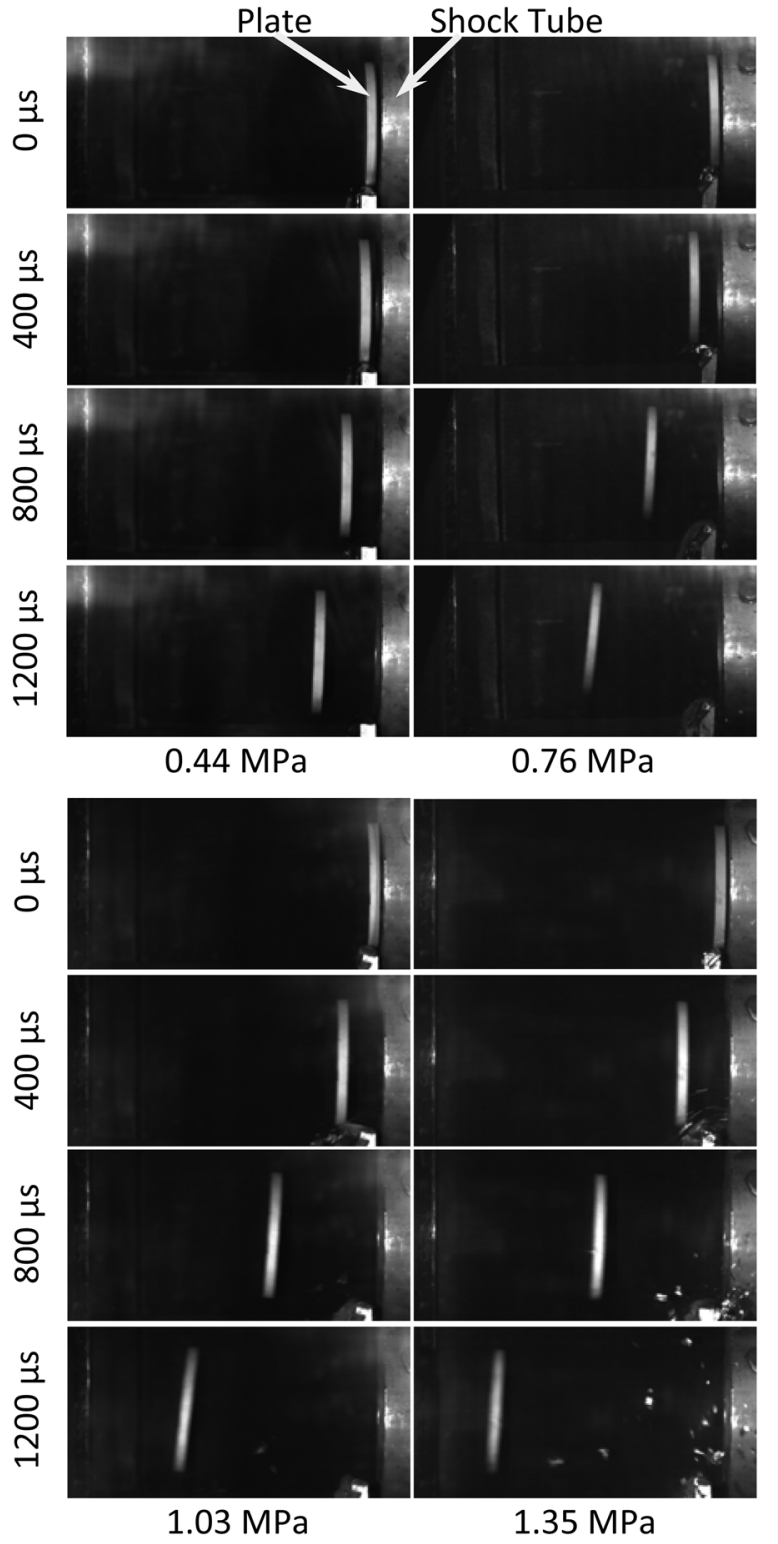

FIG. 8. High-speed side-view images of aluminum plates for different shock loadings.

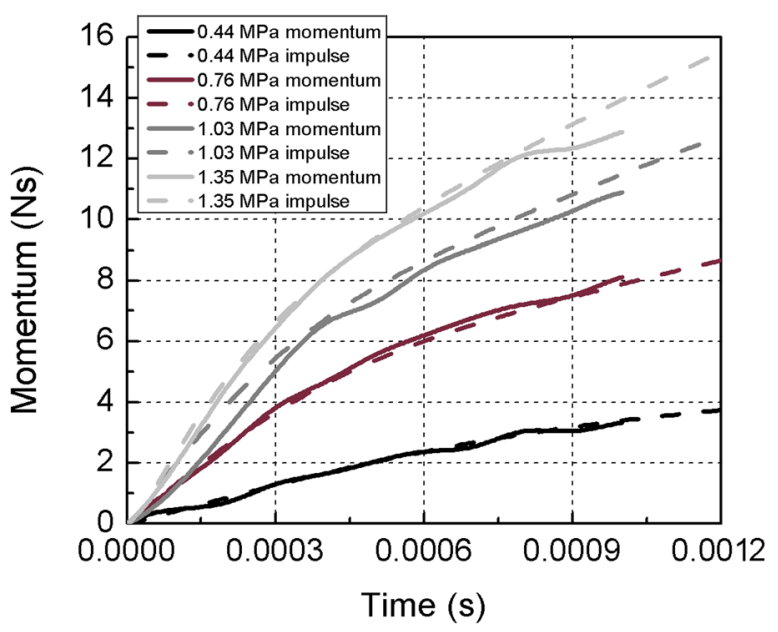

FIG. 9. (Color online) Comparison of the momentum of the plates and the impulse applied on the plates for different peak incident shock pressures. 
TABLE II. The input experimental parameters.

\begin{tabular}{lc}
\hline \hline Parameters & Value \\
\hline Pressure $p_{0}$ of the undisturbed gas & $0.101325 \mathrm{MPa}$ \\
Density $\rho_{0}$ of the undisturbed gas & $1.21 \mathrm{~kg} / \mathrm{m}^{3}$ \\
Sound velocity $c_{0}$ of the undisturbed gas & $343 \mathrm{~m} / \mathrm{s}$ \\
Particle velocity $u_{0}$ of the undisturbed gas & $0 \mathrm{~m} / \mathrm{s}$ \\
Adiabatic coefficient $\gamma$ & 1.4 \\
\hline \hline
\end{tabular}

data, while the present model gives a more accurate prediction especially at the very beginning (before $250 \mu \mathrm{s}$ ). However, after $700 \mu \mathrm{s}$, Taylor's model gives a perfect prediction. This indicates that the compressibility of the gas only has a significant effect on the pressure profile at the beginning of the shock wave loading process. Actually, the fluid-structure interaction process is almost over by this time (for example $700 \mu \mathrm{s}$ ) because the gas pressure has dropped to about $30 \%$ of its peak value, and the compressibility of the gas can be ignored. The load on the plates comes from the wind (movement of the gas particles) behind the shock wave front.

\section{The momentum transmitted onto the specimen}

The momentum transmitted into the specimen from the experiments and the results predicted by the present model and Taylor's model are shown in Fig. 13. At low peak incident pressure (for example 0.44 MPa), Taylor's model over predicts the momentum transmitted onto the plates while at high peak incident pressure (for example 1.35 MPa), it under predicts this momentum. The present model gives more accurate results than those obtained from Taylor's model especially under a shock wave loading with a high peak incident pressure (for example, 1.03 $\mathrm{MPa}$ and $1.35 \mathrm{MPa}$ ).

\section{The velocity and displacement history of the plates}

The velocity and displacement histories of the plates determined by Eqs. (49) and (50) under a shock wave loading with a peak incident pressure $1.03 \mathrm{MPa}$ are plotted as an example in Figs. 14 and 15, respectively. The prediction

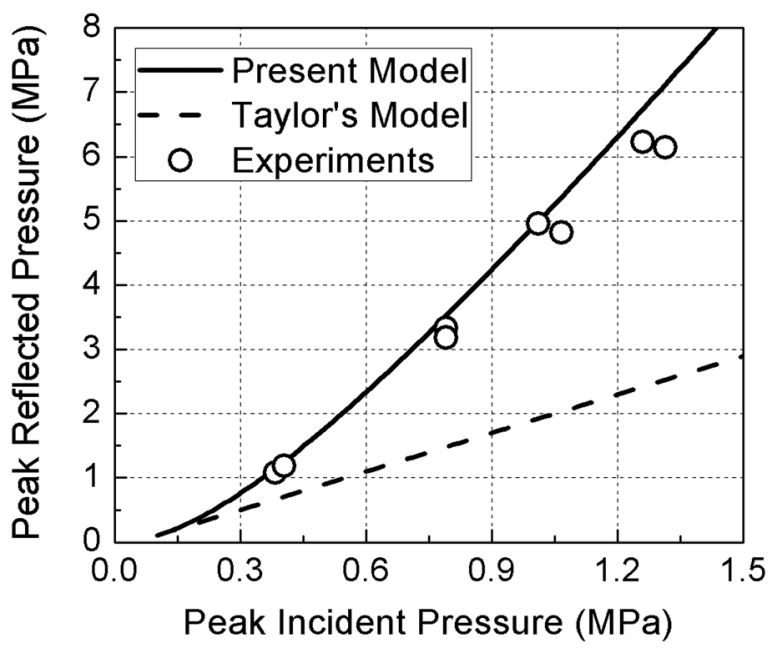

FIG. 10. The relation between peak incident and peak reflected pressures. from the present model agrees with the experimental results very well at times between 0 and about $400 \mu$ s and relatively well till about $700 \mu$ s when the peak pressure drops considerably and the compressibility of the gas can be ignored. After that time, the present model over predicts the velocity of the plate. Due to the momentum conservation, the predicted velocity from the present model approaches the experimental data at later times. The prediction of Taylor's model under predicts the velocity during the whole process.

For the displacement history plots, the prediction from the present model matches well with the experimental data until $1000 \mu$ s after which it over predicts experimental data. The Taylor's model under predicts the experimental data for all times. The difference between the prediction of the present model and the experimental data is less than $15 \%$, whereas the difference between the prediction of Taylor's model and the experimental data is higher than $25 \%$ at the time $t=3000 \mu \mathrm{s}$.

\section{Fluid structure interaction behavior in an air blast}

\section{Effect of the areal density of the plate}

As discussed in Sec. IV C 1, the areal density of a plate will only affect the positive time period $t_{2+}$ or the time constant $\theta_{2}$ of the reflected pressure and consequently affect the impulse transmitted to the plate. The effect of the areal density of a plate on the positive time period $t_{2+}$ of the reflected pressure is shown in Fig. 16. It shows that the positive time period $t_{2+}$ increases with the increase of the areal density. This means that the heavier plate will suffer longer reflected pressure duration and obtain more momentum from the blast loading. When the areal density approaches infinity, the predicted positive time period $t_{2+}$ of the reflected pressure approaches the positive time period $t_{1+}$ of the incident pressure. This indicates that when a shock wave impinges on a rigid wall, the positive time period $t_{2+}$ should be equal to the positive time period $t_{1+}$.

The effect of the areal density of a plate on the transmitted momentum is shown in Fig. 17. The effect of the areal density on the transmitted momentum is the same as the effect of the positive time period $t_{2+}$. It can be also seen that when the areal density approaches infinity, the transmitted momentums under different incident pressures all approach certain constants. These constants are defined as the maximum achievable impulse from the shock loading.

\section{Maximum achievable impulse}

The maximum achievable impulse or the maximum momentum that can be transmitted onto the structures is one of the most important parameters in evaluating the efficiency of a blast loading. This maximum impulse can only be realized when the shock wave impinges upon a rigid wall. In Taylor's model, it is calculated to be twice that of the impulse of the incident pressure. ${ }^{1,5-7}$ However, this is only valid when the fluid is incompressible or under the acoustic limit. For a fluid with high compressibility or that contains a shock wave not near the acoustic limit, the maximum achievable impulse needs to be calculated. 


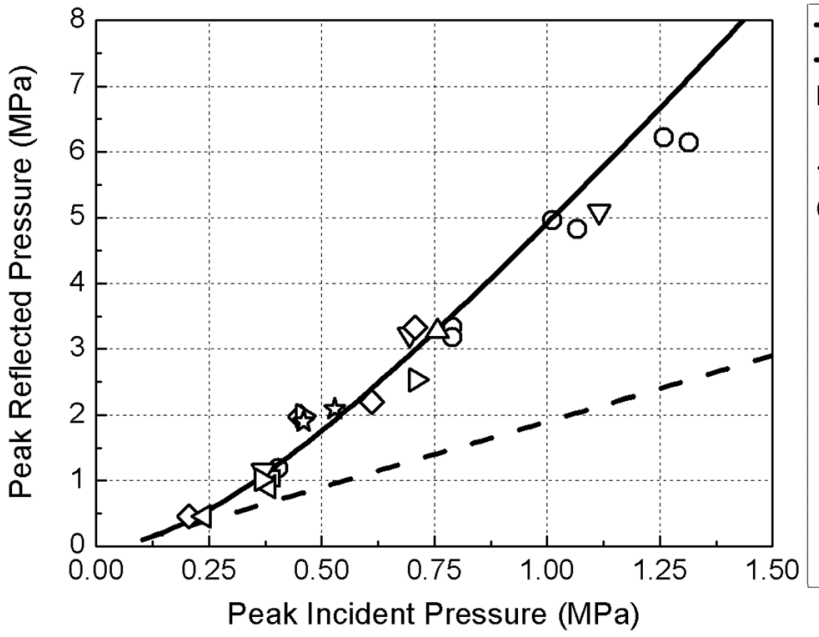

The momentum transmitted into the plate in the present model has been described in Eq. (18). The key issue is to determine the reflected pressure profile when an incident shock wave impinges upon a rigid wall. As discussed in Sec. IV C 1, Eq. (46) can be used to calculate the peak reflected pressure from a rigid wall. When a shock wave impinges on a rigid wall, the positive time period $t_{2+}$ of the reflected pressure should be equal to the positive time period $t_{1+}$ of the incident pressure (Sec. VI C 1). Therefore, the reflected pressure can be expressed as,

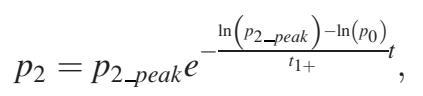

where, $p_{2 \text {-peak }}$ can be calculated from Eq. (46).

The maximum achievable impulse can then be calculated as,

$$
I_{\max }=\int_{0}^{t_{1+}} p_{2 \_ \text {peak }} e^{-\frac{t}{\theta_{2}}} d t \approx p_{2 \_ \text {peak }} \theta_{2}=\frac{p_{2 \_p e a k} t_{1+}}{\ln \left(p_{2 \text { peak }}\right)-\ln \left(p_{0}\right)} .
$$

The maximum achievable impulse evaluated from the present model and Taylor's model from the incident pressure profiles with different peak pressures, $p_{1 \_ \text {peak }}$, is shown in Fig. 18. The positive time period $t_{1+}$ is assumed to be

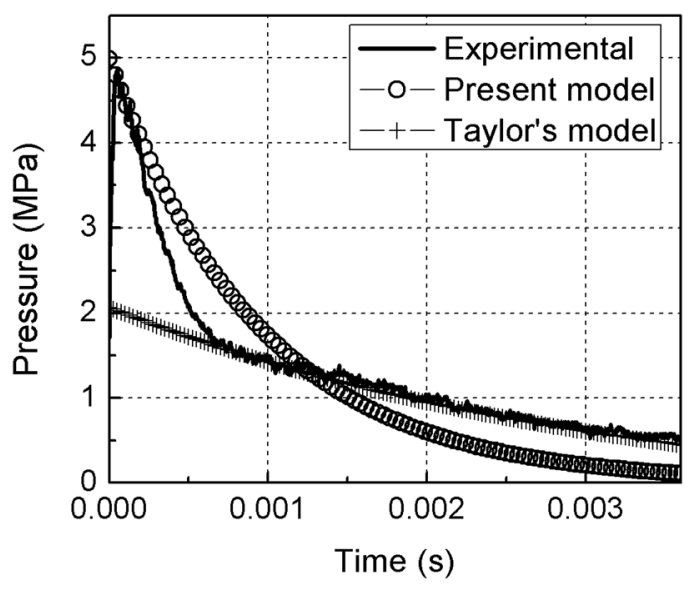

FIG. 12. Experimental and predicted reflected pressure profiles.
$0.008 \mathrm{~s}$. Two key features are evident in this figure. First, the maximum achievable impulse predicted from the present model is much higher than that predicted from the Taylor's model especially under high level incident shock wave. The difference for a $3 \mathrm{MPa}$ peak incident pressure case is larger than $200 \%$. Second, the maximum achievable impulse linearly increases with the increase of the peak incident pressure. Note that the maximum achievable impulse is linearly related to the positive time period $t_{1+}$ from Eq. (53). Therefore, the maximum achievable impulse is linearly related to the gas impulse of the incident pressure.

\section{Discussion of possible errors in present analysis and experiments}

The fluid structure interaction model developed in this study and the analysis of the experimental data make certain assumptions that could create possible errors, and this warrants some discussion. First, the exponential decay profile assumed for the reflected pulse may not exist in reality. This assumption was used because the authors felt that the

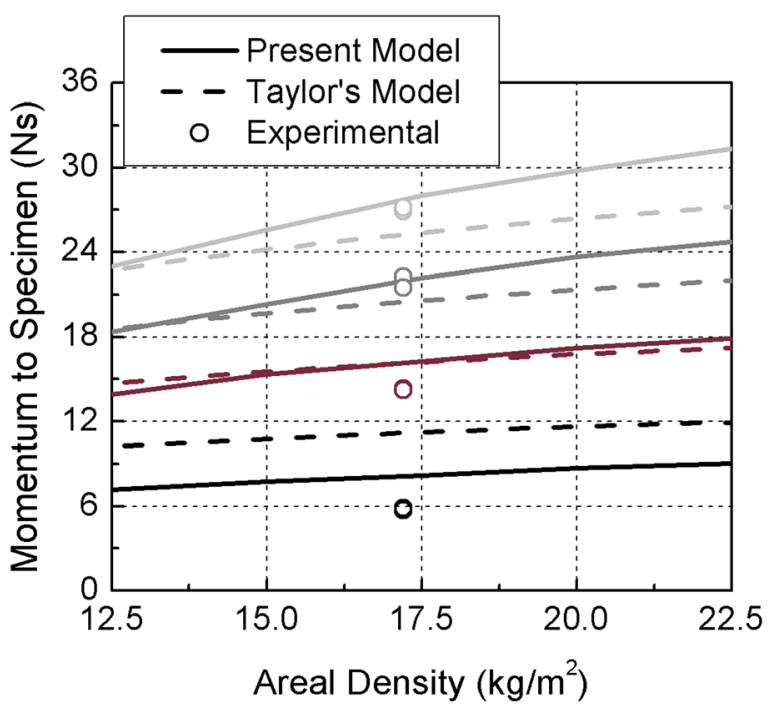

FIG. 13. (Color online) Experimental and analytical momentum transmitted onto the plates. The colors represent the different level of the incident peak pressure, black, 0.44 MPa; dark gray, $0.76 \mathrm{MPa}$; gray, $1.03 \mathrm{MPa}$; light gray, $1.35 \mathrm{Mpa}$. 


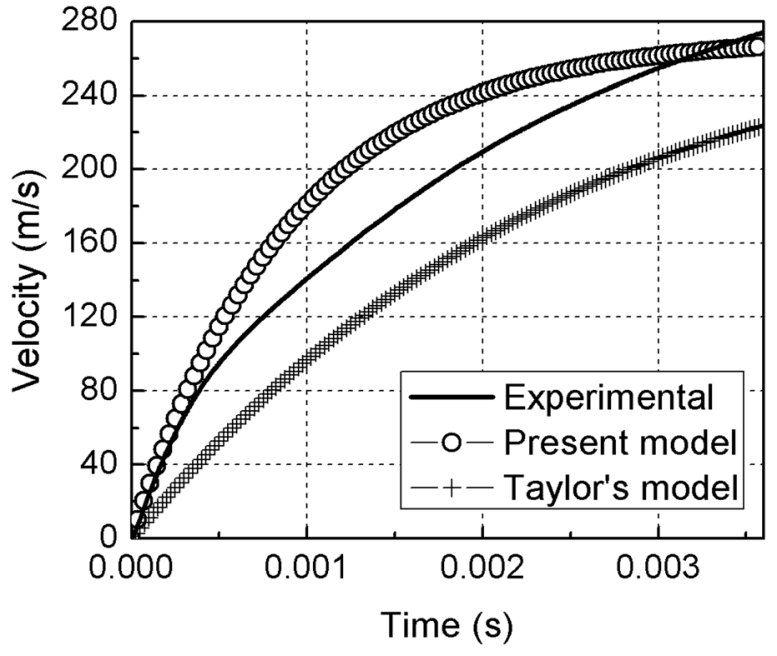

FIG. 14. Velocity history of the aluminum plate.

reflected shock wave should have a profile similar to the incident shock wave. However, the reflected pressure profiles shown in the experimental data in Fig. 7 do not display a smooth curvature during the shock loading process. They always have a turning point related to the time after which the compressibility can be ignored. The exponential decay profile cannot describe this shape. Glasstone, ${ }^{2}$ Baker et al. ${ }^{3}$ and Smith $\mathrm{et} \mathrm{al}^{4}{ }^{4}$ claimed that this turning point is related to the specimen's dimension. Second, the assumption that momentum conservation is achieved at the time when the reflected pressure decays to zero may not be completely correct. In fact, momentum conservation should be achieved during the whole shock loading process. The velocity difference between the prediction and the experiment in Fig. 13 can be improved by using a complex reflected pressure model.

In the experimental analysis, there are also two assumptions that may not be fully accurate. First, a shock tube experiment can be considered a one-dimensional shock loading experiment. Actually, this can only be achieved if the plate glides in the tube like a piston and there is no leakage of gas. However, in the current experimental setup, the plate

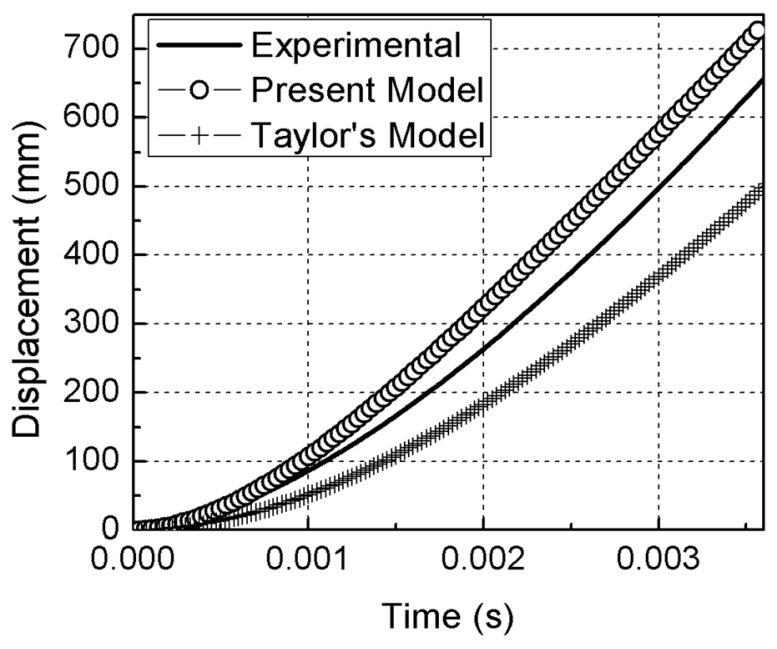

FIG. 15. Displacement-time histories of the plates.

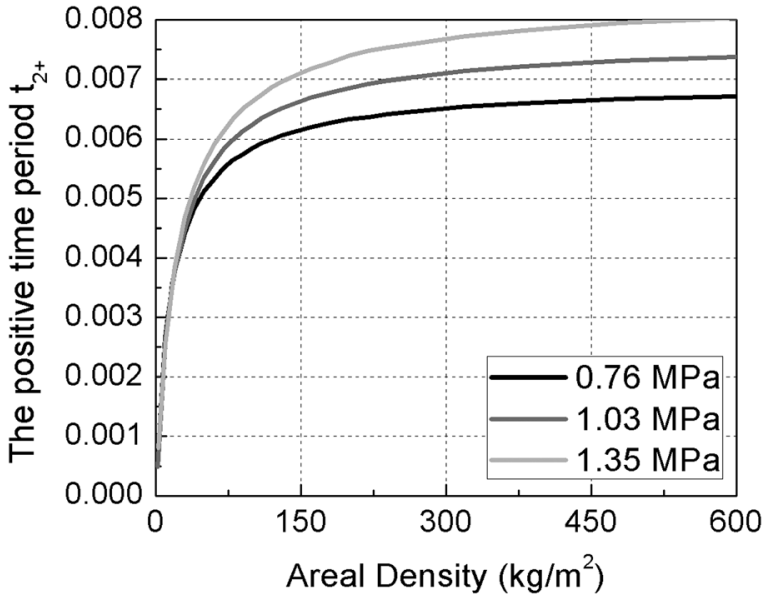

FIG. 16. Effect of the areal density on the positive time period $t_{2+}$.

flies freely from the muzzle. There is some gas leakage, and this might affect the pressure profile due to the expansion of the leaking gas. This means the measured pressure profile may be lower after some initial time than that under a real one-dimensional shock loading. The second assumption is that the measured pressure profile is the pressure profile applied on the plates. Although, Wang and Shukla ${ }^{11}$ showed that the pressure on center of a fixed specimen is the same as measured by the transducer on the shock tube, as the plates are moving in this experiment, this assumption might cause some error. When the plate moves to a position far from the muzzle (later times), the measured pressure from the pressure transducer might be lower than the real pressure applied on the plates. These effects would be more pronounced at later times ( $t$ greater than $750 \mu \mathrm{s})$ by which time the peak loads would have considerably dropped and could be the reason for the differences between the model's prediction and the experimental results.

\section{SUMMARY}

A new fluid structure interaction model, which considers the compressibility of a gas, is proposed and implemented in

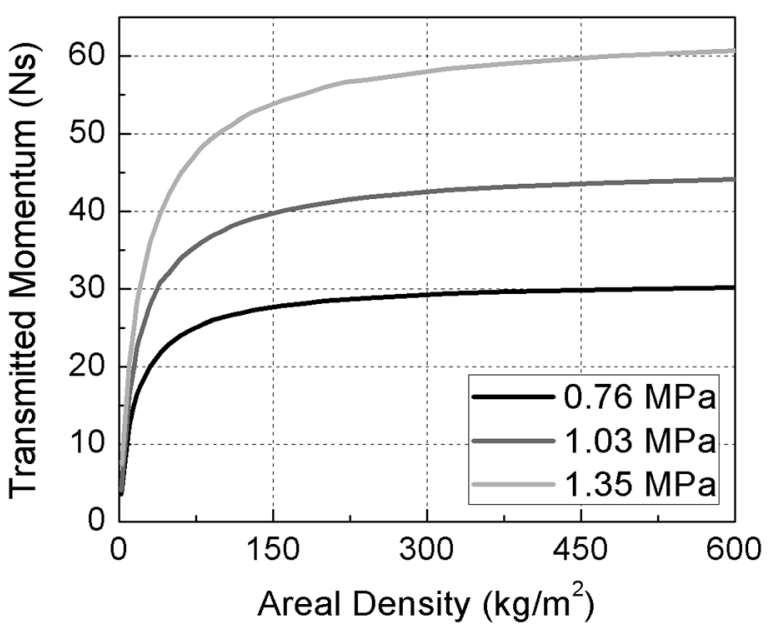

FIG. 17. Effect of the areal density on the transmitted momentum. 


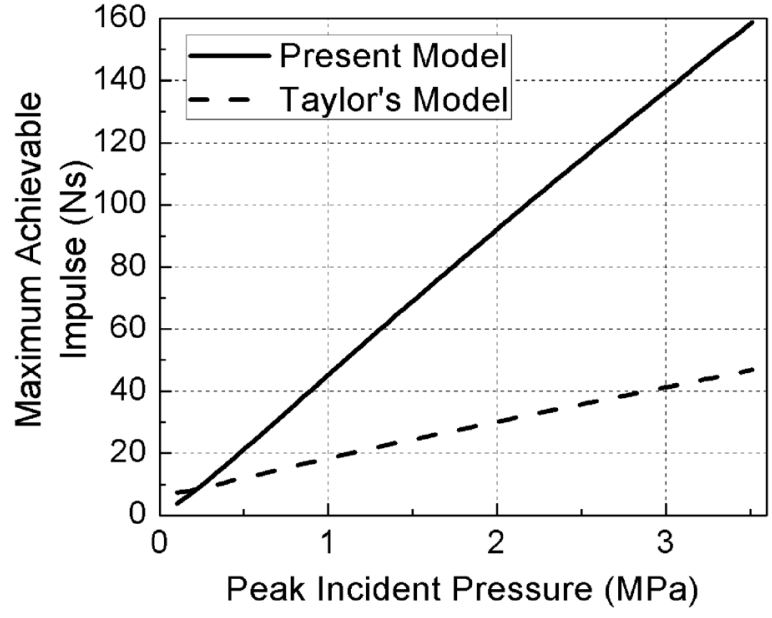

FIG. 18. Evaluated maximum achievable impulse comparison.

this study. One-dimensional gas-dynamic theory (the Rankine-Hugoniot relation) for an ideal gas was utilized to consider the compressibility of the gas and evaluate its physical parameters such as density, particle velocity, and sound velocity in the gas. The conservation between the pressure impulse applied on the plate and the momentum of the plate itself was utilized to determine the reflected pressure profile. A series of shock wave loading experiments on free-standing monolithic aluminum plates was conducted using a shock tube apparatus. The measured reflected pressure profiles and the momentum transmitted onto the plates were compared with the results predicted by the present model and previous models. ${ }^{1,5-9}$ The present model predicts more accurate results for the peak reflected pressure, the reflected pressure profile, the momentum transmitted onto the plate, and the motion of the plate. Further analysis shows that the peak reflected pressure is only related to the peak incident pressure; this is confirmed by the experimental results. The increase in the areal density of a plate causes an increase in the positive time period of the reflected pressure and the total momentum transmitted into the plate.

\section{ACKNOWLEDGMENTS}

The authors kindly acknowledge the financial support of the Office of Naval Research (Dr. Y.D.S. Rajapakse) under Grant No. N000140410268 and the Department of Homeland Security under Cooperative Agreement No. 2008-ST-061ED0002.

${ }^{1}$ G. I. Taylor, in The Scientific Papers of Sir Geoffrey Ingram Taylor. Aerodynamics and the Mechanics of Projectiles and Explosions, edited by G. K. Batchelor (Cambridge University Press, Cambridge, UK, 1963), Vol. III, pp. 287-303.

${ }^{2}$ S. Glasstone, The Effects of Nuclear Weapons (U.S. Government Printing Office, Washington, DC, 1964).

${ }^{3}$ W. E. Baker, P. A. Cox, P. S. Westine, J. J. Kulesz, and R. A. Strehlow, Explosion Hazards and Evaluation (Elsevier, New York, 1983).

${ }^{4}$ P. D. Smith and J. G. Hetherington, Blast and Ballistic Loading of Structures (Butterwirth-Heinemann Elsevier Science, Oxford, UK, 1994).

${ }^{5}$ Z. Xue and J. W. Hutchinson, Int. J. Impact Eng. 30, 1283-1305 (2004).

${ }^{6}$ N. A. Fleck and V. S. Deshpande, J. Appl. Mech. 71, 386-401 (2004).

${ }^{7}$ V. S. Deshpande and N. A. Fleck, J. Mech. Phys. Solids. 53, 2347-2383 (2005).

${ }^{8} \mathrm{~N}$. Kambouchev, Ph.D dissertation, Massachusetts Institute of Technology, 2007.

${ }^{9}$ N. Kambouchev, L. Noels, and R. Radovitzky, J. Appl. Phys. 100, 063519 (2006).

${ }^{10}$ P. J. Tan, S. R. Reid, and J. J. Harrigan, J. Appl. Mech. 72, 978-979 (2005).

${ }^{11}$ E. Wang and A. Shukla, Int. J. Impact Eng. 37, 1188-1196 (2010).

${ }^{12}$ G. N. Nurick, G. S. Langdon, Y. Chi, and N. Jacob, Comp. Struct. 91, 433-441 (2009).

${ }^{13}$ J. Shen, G. Lu, Z. Wang, and L. Zhao, Int. J. Impact Eng. 37, 960-970 (2010).

${ }^{14}$ R. Courant and K. O. Friedrichs, Supersonic Flow and Shock Waves (Interscience Publishers, New York, 1948).

${ }^{15}$ R. A. Granger, Fluid Mechanics (Dover Publications, Inc., Mineola, NY, 1985).

${ }^{16}$ See the webpage: http://aerorocket.com/Nozzle/Validate/Tshock/Shock.html for information about the thickness of the shock wave front.

${ }^{17}$ J. LeBlanc, A. Shukla, A. C. Rousseau, and A. Bogdanovich, Comp. Struct. 79, 344-355 (2007).

${ }^{18}$ A. Shukla, G. Ravichandran, Y. D. S. Rajapakse, Dynamic Failure of Materials and Structures (Springer, New York, 2009).

${ }^{19}$ M. Stoffel, R. Schmidt, and D. Weichert, Int. J. Solid Struct. 38, 7659-7680 (2001).

${ }^{20}$ E. Wang, N. Gardner, and A. Shukla, Int. J. Solid Struct. 46, 3492-3502 (2009).

${ }^{21}$ E. Wang and A. Shukla, Exp. Mech. (2011).

${ }^{22}$ S. A. Tekalur, Ph.D dissertation, University of Rhode Island, 2007. 